\title{
Characteristics of Quasi Microwave-Optical Single-Sideband Signal Generation Using a Nonlinear Semiconductor Optical Amplifier
}

\author{
Minho Park ${ }^{*}$ and Ju-Hang Sohn \\ Agency for Defense and Development for Korea, 31486, Korea \\ mhpark2@add.re.kr,spson1@add.re.kr
}

\begin{abstract}
The characteristics of a quasi microwave-optical single-sideband (qMOSSB) signal generated using a nonlinear semiconductor optical amplifier (NSOA) were studied. Dependence on the polarization and chirp of the NSOA input optical signal, the power and frequency of the EAM input radio frequency $(R F)$ signal, and the NSOA bias current on the characteristics of the qMOSSB signal was investigated by measuring the difference of the sideband optical powers of the qMOSSB signal
\end{abstract}

Keywords: Radio-over-fiber communication, fiber dispersion, quasi microwave-optical single-sideband (qMOSSB), polarization, chirp

\section{Introduction}

The radio-over-fiber (RoF) system is a well-known link for broadband wireless and wired communication networks [1]. Single-mode fiber (SMF), which is used as a medium for transmission of radio frequency (RF) modulated optical signals in RoF systems, has ultra-low loss and ultra-wide bandwidth properties suitable for broadband microwave applications. However, the chromatic dispersion of SMF introduces fluctuation of the RF signal power at photodetector when RF-modulated optical signals in the form of an optical double-sideband (ODSB) signal are received [2]. The use of RF-modulated optical signals in the form of an optical single sideband (OSSB) signal can alleviate the power fading problem, and there have been many reports on generation of OSSB signals [2-6]. Among them, methods using an optical filter [3] and a dual-electrode Mach-Zehnder modulator (DEMZM) [2] are well known. An optical filter is used to eliminate one of the sidebands in an ODSB signal to generate an OSSB signal. This method, however, requires a filter with stabilized and narrow bandwidth characteristics and has difficulty in scalability when wavelength-division multiplexing (WDM) is employed. The DEMZM is typically implemented with lithium niobate ( $\mathrm{LiNbO} 3)$ and thus cannot be integrated with other semiconductor devices, including laser diodes (LDs), semiconductor optical amplifiers (SOAs), and so on. It also requires a higher operating power than other semiconductor-based modulators.

Recently, a generating method of quasi microwave-optical single-sideband (qMOSSB) signals using a nonlinear semiconductor optical amplifier (NSOA) was known [7, 9]. The qMOSSB signal was generated by transformation of an ODSB signal generated by an electroabsorption modulator (EAM). The coherent population oscillation (CPO) effects in an NSOA modify the magnitude and phase of two sidebands of the input ODSB signal, generating a qMOSSB signal. The qMOSSB signal had a larger red-shifted sideband power than that of a blue-shifted sideband. When transmitted over SMF, the qMOSSB signal showed a reduction in the power fading problem associated with fiber dispersion.

Received (October 6, 2017), Review Result (December 19, 2017), Accepted (December 26, 2017)

* Corresponding Author 
The qMOSSB signal generation using an NSOA has a possibility of integration with other semiconductor-based photonic devices and scalability for WDM applications. In this paper, dependence on several values with respect to the chirp and polarization of the ODSB signal, the power and frequency of the EAM input RF signal, and the NSOA bias on the characteristics of the qMOSSB signal was investigated.

\section{Effect of the Chirp Induced by the EAM}

Figure 1 represents the experimental setup to study the influence of chirp in the ODSB signal generated by an EAM on the difference of the qMOSSB signals. The input RF power to the EAM (OM5642-W-30B) was fixed at $0 \mathrm{dBm}$. The Erbium doped fiber amplifier (EDFA) and variable optical attenuator (VOA) were used to set the VOA output power to $0 \mathrm{dBm}$. The polarization of the ODSB signal generated by the EAM was set to $+45^{\circ}$ linear polarization (LP) state by the polarization controller (PC). The NSOA (SOANL-OEC-1550) was biased at the current of $350 \mathrm{~mA}$. The chirp values were characterized using the method presented in [8] with respect to three different laser diode (LD) wavelengths $(1530,1550$, and $1570 \mathrm{~nm})$. The optical spectrum of an qMOSSB signal generated by the NSOA were measured by an optical spectrum analyzer (OSA).

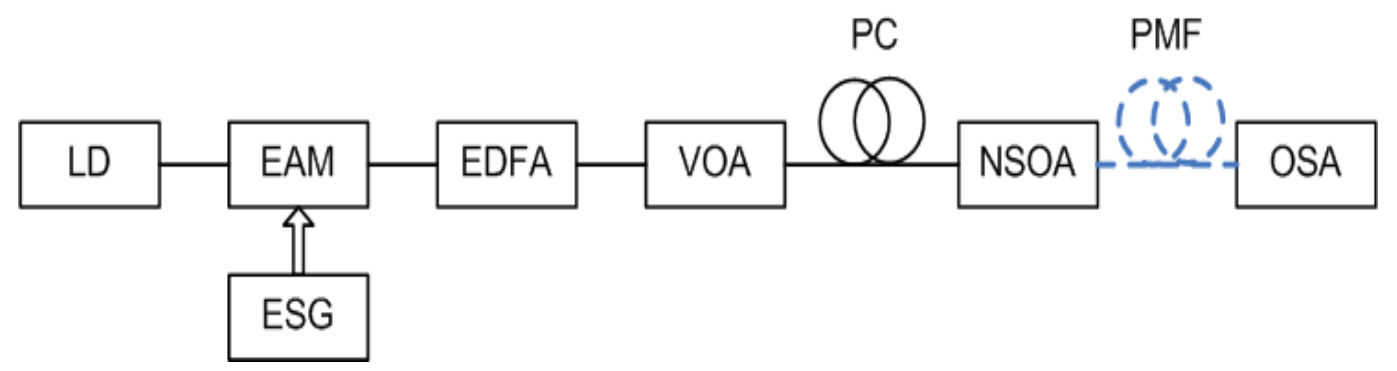

Figure 1. Experimental Setup for Investigation of the Effect of the EAM Chirp on the Disparity of Sidebands of the Qmossb Signal. LD: Laser Diode, EAM: Electroabsorption Modulator, EDFA: Erbium-Doped Fiber Amplifier, VOA: Variable Optical Attenuator, PC: Polarization Controller, NSOA: Nonlinear Semiconductor Optical Amplifier, PMF: Polarization Maintaining Fiber, OSA: Optical Spectrum Analyzer, ESG: Electrical Signal Generator

The difference of sidebands (defined as the ratio of the power of the red-shifted sideband to that of the blue-shifted sideband) of the qMOSSB signal and the EAM chirp value as functions of the EAM bias voltage for different LD wavelengths and RF frequencies are presented in Figure 2. It was observed that the zero chirp bias of the EAM became more negative as the wavelength of the LD was increased. It was also observed that the disparity of sidebands of the qMOSSB signal was maximized when the EAM was set at the zero chirp bias voltage for three radio frequencies $(33,35,37 \mathrm{GHz})$ and $\mathrm{LD}$ wavelengths investigated.

The effect on the optical power of sidebands of the qMOSSB signal was measured with respect to the EAM bias. Figure 3 shows the optical power of one of the sidebands of the ODSB signal and the optical power gap of sidebands of the qMOSSB signal as a function of the electroabsorption modulator bias at the LD wavelength of $1550 \mathrm{~nm}$ and the RF frequency of $35 \mathrm{GHz}$. As the EAM bias became more negative, the optical power of sidebands of the ODSB signal elevated and then saturated. However, the optical power gap of sidebands of the qMOSSB signal increased, maximized and reduced as the EAM bias became more negative and then reached the maximum value when the EAM was biased at the zero chirp bias point $(-2.5 \mathrm{~V})$. 


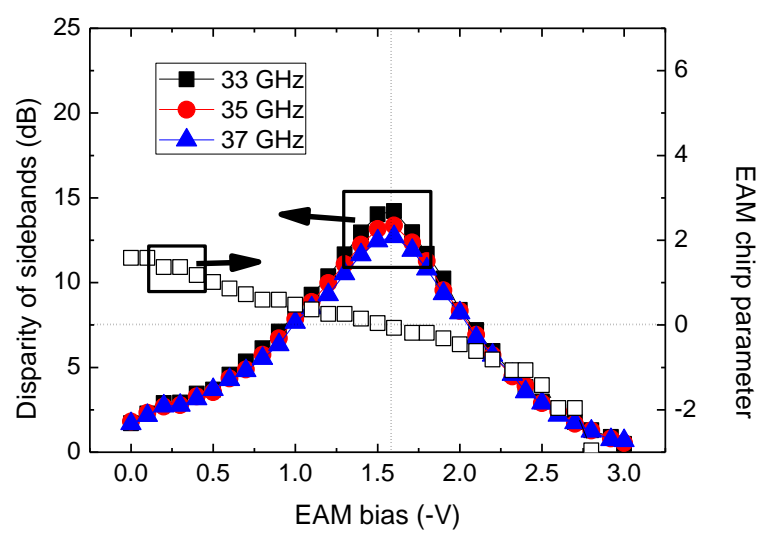

(a) LD wavelength of $1530 \mathrm{~nm}$

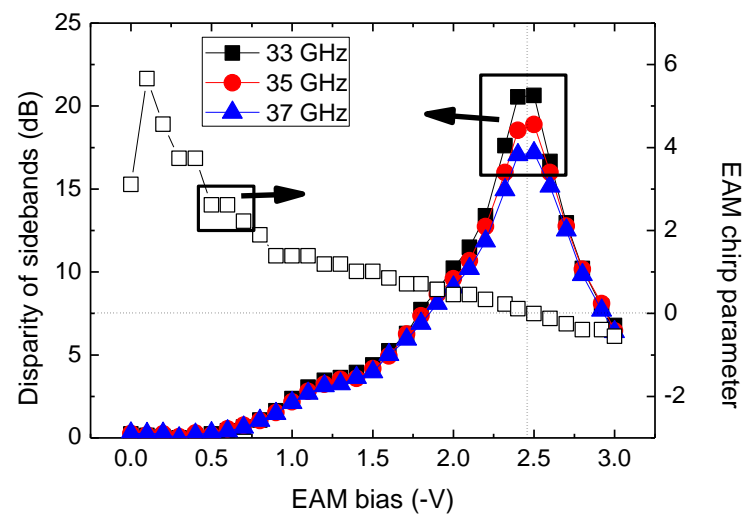

(b) LD wavelength of $1550 \mathrm{~nm}$

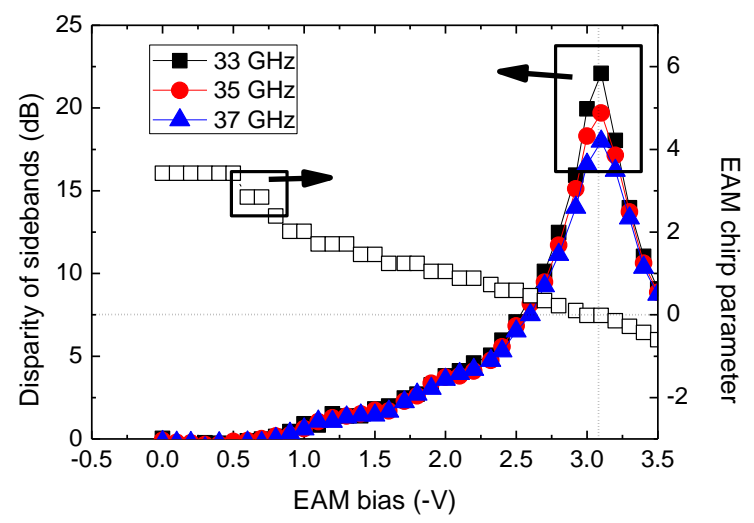

(c) LD wavelength of $1570 \mathrm{~nm}$

Figure 2. Measured Disparity of Sidebands of the qMOSSB Signal and EAM Chirp Parameters as a Function of the EAM Bias for Various LD Wavelengths and RF Frequencies 


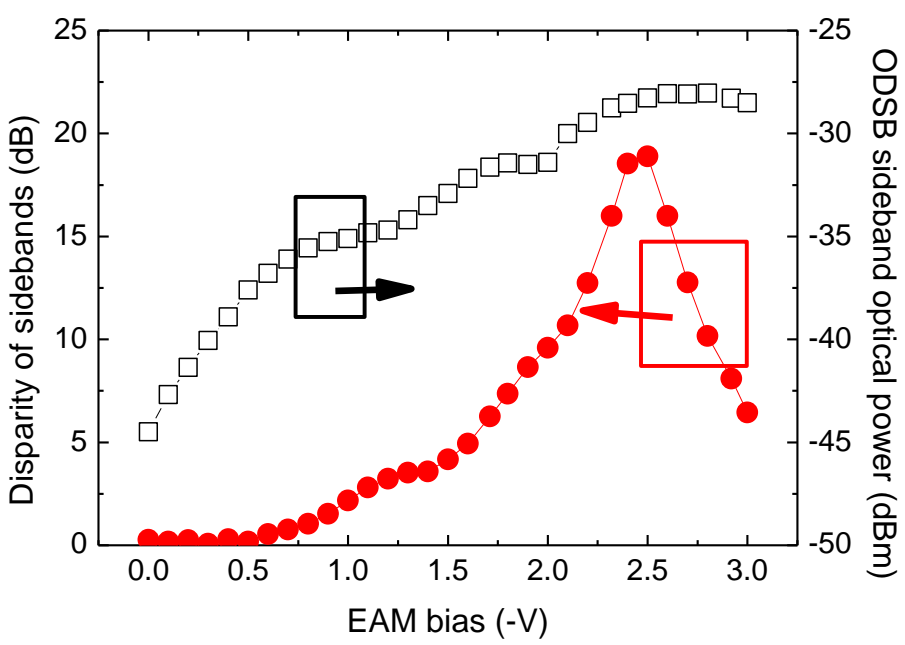

Figure 3. Disparity of Sidebands of the qMOSSB Signal and Optical Power of the Sideband of the ODSB Signal as a Function of the EAM Bias for the LD Wavelength of $1550 \mathrm{~nm}$ and the RF Frequency of $35 \mathrm{GHz}$

\section{Effect of the Polarization of the Input ODSB signal}

The experimental setup for investigation of the effect of the polarization of the input ODSB signal on the qMOSSB signal generation is shown in Figure 4. The output of a distributed feedback laser diode (DFB LD) with a wavelength of $1550 \mathrm{~nm}$ was modulated by an EAM with a $35 \mathrm{GHz}$ RF signal to generate an ODSB signal. The power of the RF signal was $0 \mathrm{dBm}$, and the EAM was biased at a zero chirp point. The power of the ODSB signal was controlled by a VOA so that the input optical power to the NSOA could be set to $-10 \mathrm{dBm}$. The polarization of the ODSB signal was controlled by the PC. A part of the ODSB signal was taken by a polarization maintaining optical coupler (PMOC) and sent to a state of polarization (SOP) analyzer (Fiberpro, SA2000) through a polarization maintaining fiber (PMF) to measure the polarization state (i.e., the Stoke parameters). The other branch of the PMOC was directed to the NSOA through a PMF for the qMOSSB signal generation. The generated qMOSSB signal was then directed to an OSA to analyze the sidebands of the qMOSSB signal.

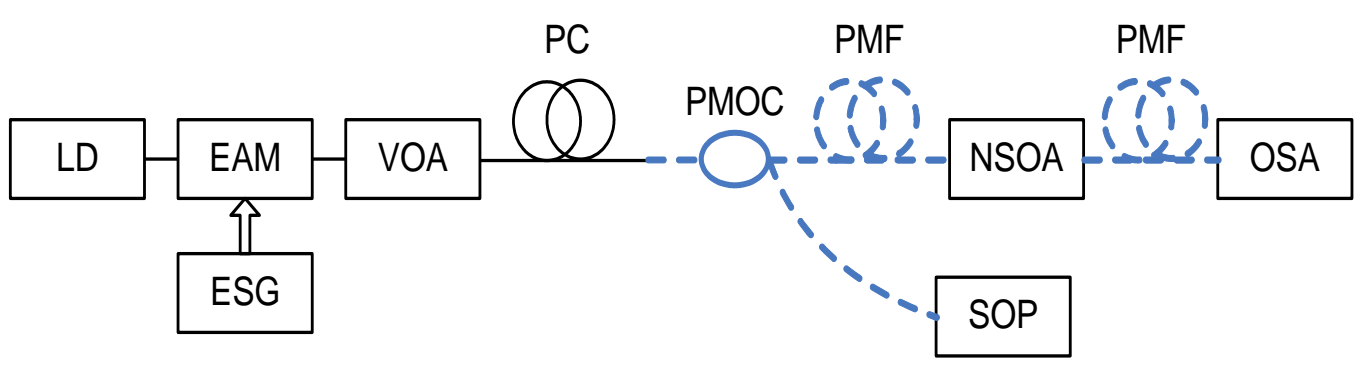

Figure 4. Experimental Setup for Characterization of the Effect of the Polarization of the Input Optical Signal on a qMOSSB Signal. PMOC: Polarization Maintaining Optical Coupler, SOP: State of Polarization Analyzer

The disparity of the sidebands of the qMOSSB signal for various polarization states of the input ODSB signal is shown in Figure 5. The disparity of the sidebands became the 
smallest when the Stoke parameter S3 was 1 and the Stoke parameter S1 was close to 0. The Stoke parameter S3 value of approximately 1 means that the polarization of the input ODSB signal was close to the right circular polarization (RCP). The disparity of the sidebands became the largest when the S2 value was close to 1. The Stoke parameter S2 value of approximately 1 means that the polarization was close to $+45^{\circ} \mathrm{LP}$. The variation of the disparity of the sidebands of the qMOSSB signal was approximately $6 \mathrm{~dB}$ for the polarization states of the input ODSB signal investigated.

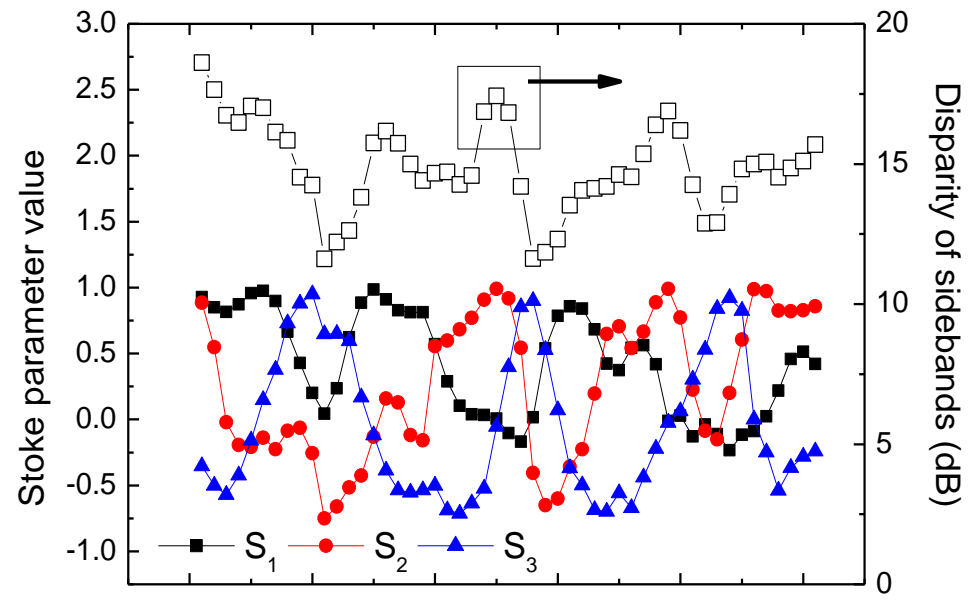

\section{Figure 5. Effect of the Polarization of the Input ODSB Signal (Stoke} Parameters) on the Disparity of the Sidebands of the qMOSSB Signal

Measuring program is transmitted and received and a spectrum analyzer data through the USB interface, and receives the GPS information from the external location information showing that driven by the program. Figure 4 is information about each of the free space loss model (applied in accordance with the propagation distance attenuation), the diffraction loss model (Non LoS obstructions environment applicable), part path loss attenuation model (Near LoS obstructions environment applicable). In consideration of the propagation environment of radio waves that target only the broadband measurements and characteristics it may be applied to terrestrial propagation model as described above[8].

\section{Effect of Power and Frequency of the EAM Input RF Signal}

The effects of the power and frequency of the EAM input RF signal on the power of the sideband of the ODSB signal and the disparity of sidebands of the qMOSSB signal were also investigated. The experimental setup is shown in Figure 6. The wavelength of the LD was $1550 \mathrm{~nm}$. The EAM was biased at the zero chirp point $(-2.5 \mathrm{~V})$. The power of the EAM input RF signal with the frequency of $35 \mathrm{GHz}$ was varied from -10 to $10 \mathrm{dBm}$. The polarization of the ODSB signal generated by the EAM was set to $+45^{\circ} \mathrm{LP}$ state by a PC. The EDFA and VOA were used to set the power of the optical carrier of the ODSB signal to $0 \mathrm{dBm}$. The NSOA bias current was $350 \mathrm{~mA}$. 


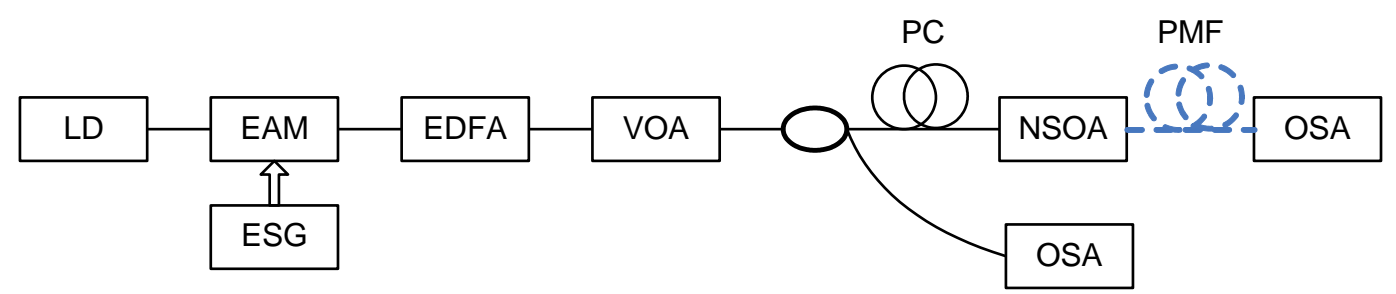

Figure 6. Experimental Setup for Investigation of the Effects of Power and Frequency of the EAM Input RF Signal on the Power of the Sideband of the ODSB Signal and the Disparity of Sidebands of the qMOSSB Signal

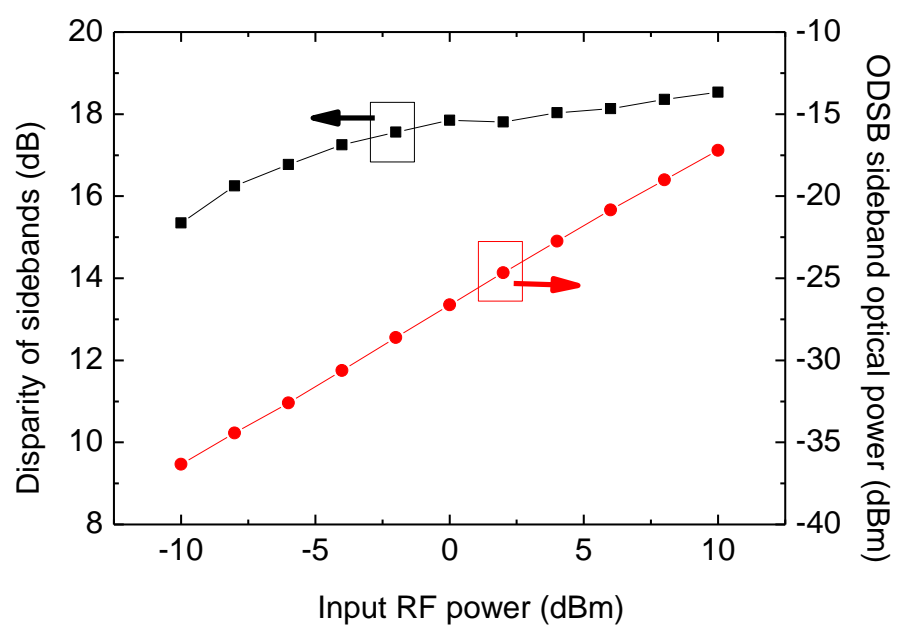

\section{Figure 7. Disparity of Sidebands of the qMOSSB Signal and Optical Power of the Sideband of the ODSB Signal as a Function of the Power of the EAM input RF Signal}

Figure 7 shows the measured disparity of the sidebands of the qMOSSB signal and the optical power of the sideband of the ODSB signal as a function of the power of the EAM input RF signal. As the power of the EAM input RF signal was changed from -10 to 10 $\mathrm{dBm}$, the ODSB sideband power changed almost linearly from -36 to $-16 \mathrm{dBm}$. On the other hand, variation of the disparity of the sidebands of the qMOSSB signal remained within $4 \mathrm{~dB}$, showing less dependence on the power of the EAM input RF signal.

The dependence of the disparity of the sidebands of the qMOSSB signal on the frequency of the EAM input signal was also investigated in a similar way. Except for the power of the EAM input RF signal, which was set to $0 \mathrm{dBm}$, the same experimental parameters were used. Figure 8 shows the disparity of the sidebands of the qMOSSB signal and the optical power of one of the sidebands of the ODSB signal as a function of the frequency of the EAM input RF signal. The optical power of the sideband of the ODSB signal was reduced by about $20 \mathrm{~dB}$ as the frequency of the EAM input signal was increased from 5 to $45 \mathrm{GHz}$, following the transfer characteristics of the EAM. The disparity of the sidebands of the qMOSSB signal increased as the frequency of the EAM input signal was increased and then reached the maximum value of approximately $18 \mathrm{~dB}$ at the RF frequency of around $30 \mathrm{GHz}$. 


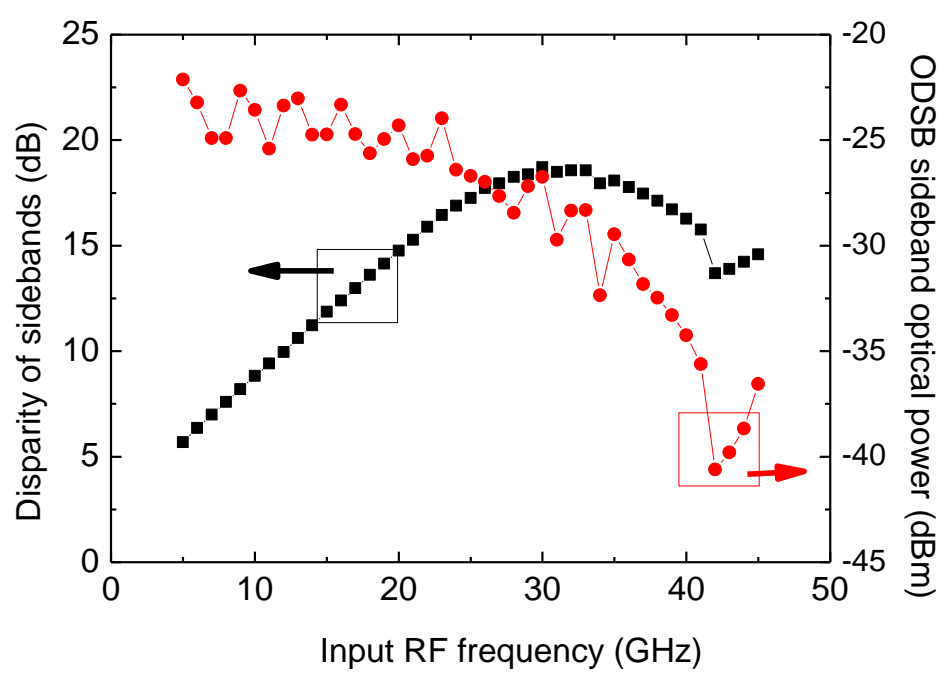

Figure 8. Disparity of Sidebands of the qMOSSB Signal and Optical Power of the Sideband of the ODSB Signal as a Function of the Frequency of the EAM Input RF Signal

\section{The NSOA Bias Current Characteristic}

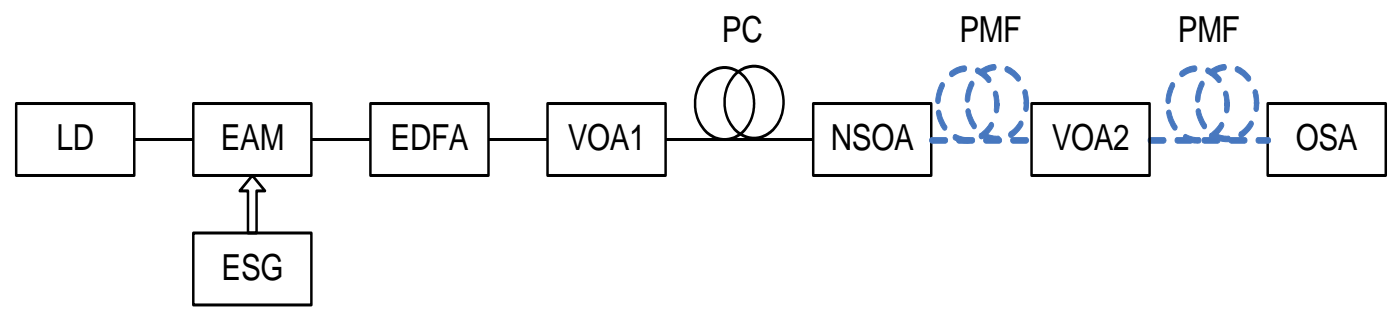

Figure 9. Experimental Setup for Investigation of the Effect of the NSOA Bias Current on the Disparity of the Sidebands of the qMOSSB Signal

The effect of the NSOA bias current on the sidebands of the qMOSSB signal was investigated using the experimental setup shown in Figure 9. The wavelength of the LD was $1550 \mathrm{~nm}$, and the input RF power to the EAM was set to $0 \mathrm{dBm}$. The EDFA and VOA1 were used to set the input optical power to the NSOA to $-10 \mathrm{dBm}$. The polarization of the ODSB signal generated by the EAM was set to $+45^{\circ} \mathrm{LP}$ state by the PC. VOA 2 was used to set the power of the carrier signal of the NSOA output to $-10 \mathrm{dBm}$ for various NSOA bias currents. 


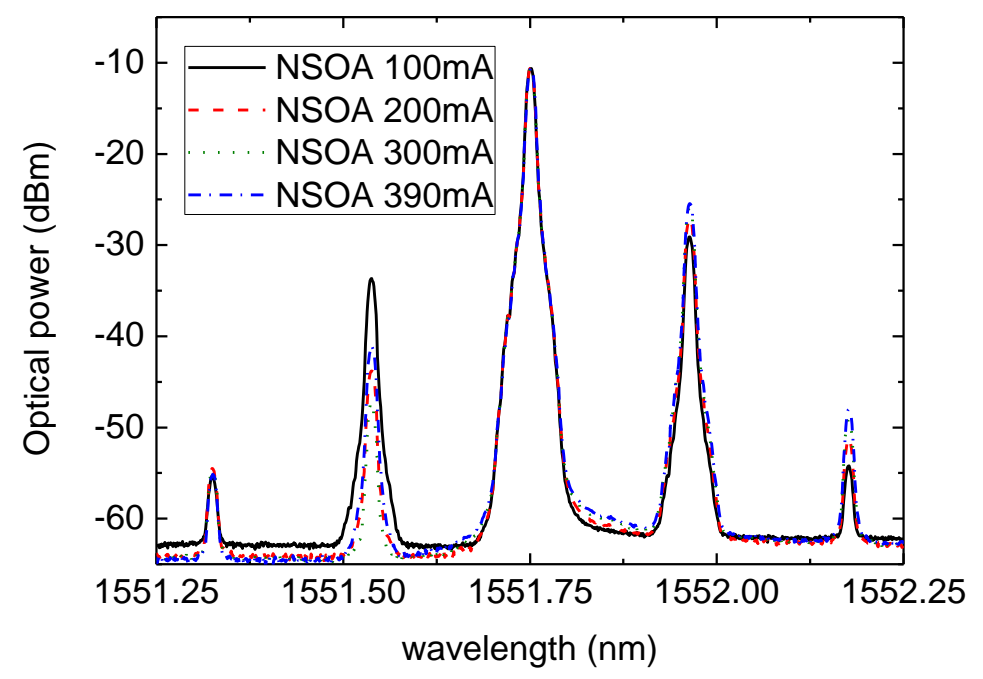

\section{Figure 10. Optical Spectra of the qMOSSB Signal for Various NSOA Bias Currents}

Figure 10 represents the optical power spectra of the qMOSSB signal as various NSOA bias currents. As can be seen in the spectra (Figure 10), the CPO effects, which change the amplitude of the blue- and red-shifted optical sidebands, are related with the NSOA bias current. Figure 11 shows the optical sidebands power and difference of the sidebands of the qMOSSB signal as a function of the NSOA bias current. At the bias currents under $50 \mathrm{~mA}$, the CPO effects in the NSOA were negligible. As the NSOA bias current was elevated, the disparity of the sidebands of the qMOSSB signal increased, reaching $25 \mathrm{~dB}$ (the maximum value) at the NSOA bias current of $250 \mathrm{~mA}$. More growth in the NSOA bias current beyond $250 \mathrm{~mA}$ decreased the gap of the sidebands of the qMOSSB signal due to the reduced suppression of the blue-shifted sideband power.

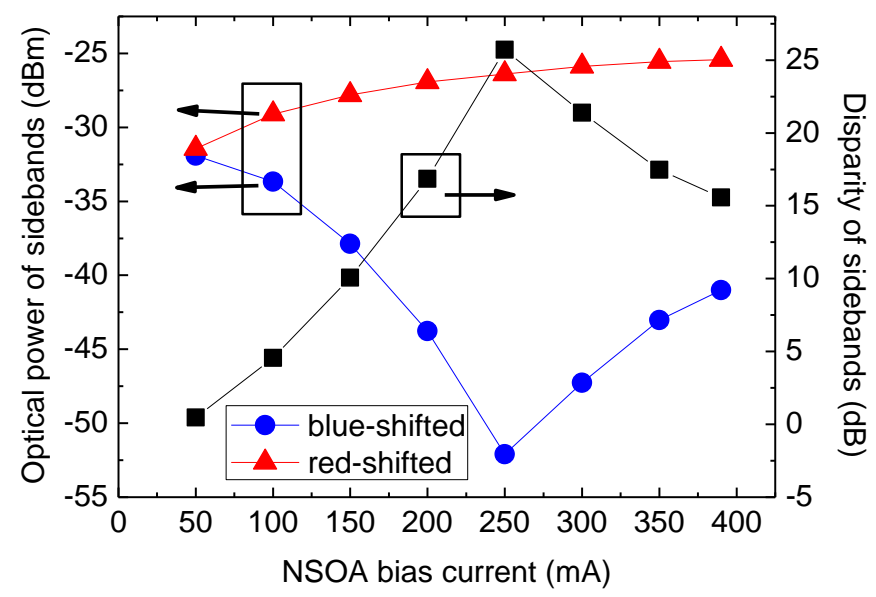

Figure 11. Measured Optical Power and Disparity of Sidebands of the qMOSSB Signal as a Function of the NSOA Bias Current 


\section{Conclusion}

The optimal parameters of the qMOSSB signal generated using an NSOA were studied. Characteristics of the chirp and polarization of the NSOA input optical signal, the power and frequency of the EAM input RF signal, and the NSOA bias current on the characteristics of the qMOSSB signal were investigated. The disparity of the blue- and red-shifted sidebands of the qMOSSB signal was dependent on the chirp value of the EAM and was maximum when the EAM was biased at a zero chirp bias point regardless of the wavelength of the LD and the frequency of the EAM input RF signal. The disparity of the sidebands of the qMOSSB signal was maximum when the polarization of the ODSB signal was +45 degrees LP state and minimum when the polarization of the ODSB signal was RCP state. The disparity was dependent on the power and frequency of the EAM input RF signal as well as the NSOA bias current.

\section{Acknowledgments}

This paper is revised and expanded version of a paper entitled 'Optimal Quasi-Optical Single-Sideband Signal Generation Using a Nonlinear Semiconductor Optical Amplifier' presented at the 4th International Conference on Green and Smart Technology 2017 by Minho Park and Ju-Hang Sohn, at Jeju, Republic of Korea (ROK) on the $1^{\text {st }}$ December, 2017.

\section{References}

[1] S. R. Abdollahi, H. S. Al-Raweshidy, R. Nilavalan and A. Darzi, "Future broadband access network challenges", Proc. 2010 17th International Conf. on Wireless and Optical Communications Networks (WOCN), (2010), pp. 1-5.

[2] G. H. Smith, D. Novak and Z. Ahmed, "Overcoming chromatic-dispersion effects in fiber-wireless systems incorporating external modulators", IEEE Trans. Microw. Theory Tech., vol. 45, no. 8, (1997), pp. 1410-1415.

[3] J. Park, W. V. Sorin and K. Y. Lau, "Elimination of the fibre chromatic dispersion penalty on 1550nm millimeter-wave optical transmission", Electron. Lett., vol. 33, (1997), pp. 512-513.

[4] H. Kim, "EML-based optical single sideband transmitter", IEEE Photonics Technol. Lett., vol. 20, no. 4, (2008), pp. 243-245.

[5] M.-T. Zhou, A. B. Sharma, Z.-H. Shao and M. Fujise, "Optical single-sideband modulation at $60 \mathrm{GHz}$ using electro-absorption modulators", in Proc. MWP 2005, (2005), pp. 121-124.

[6] H.-J. Kim and J.-I. Song, "All-optical single-sideband upconversion with an optical interleaver and a semiconductor optical amplifier for radio-over-fiber application”, Opt. Express., vol. 17, no. 12, (2009), pp. 9810-9817.

[7] M. Park, K.-C. Kim and J.-I. Song, "Generation and transmission of a quasi-optical single sideband signal for radio-over-fiber systems", IEEE Photonics Technol. Lett., vol. 23, no. 6, (2011), pp. 383-385.

[8] F. Devaux, Y. Sorel and J. F. Kerdiles, "Simple measurement of fiber dispersion and of chirp parameter of intensity modulated light emitter", J. Lightwave Technol., vol. 11, no. 12, (1993), pp. 1937-1940.

[9] M. Park, J.-H. Sohn and J.-I. Song, "Optimal Quasi-Optical Single-Sideband Signal Generation Using a Nonlinear Semiconductor Optical Amplifier", The 4th International Conference on Green and Smart Technology 2017, (2017), pp. 42-44.

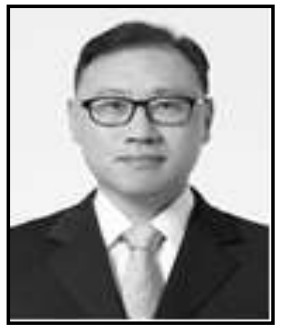

Minho Park, he received the B.S. degree in Information and Communications Engineering from Chungnam National University, Daejeon, Korea, in 2003. The M.S. and Ph. D. degrees are obtained from Gwangju Institute of Science and Technology (GIST), Gwangju, Korea, in the Department of Information and Communications, in 2005 and 2012 respectively. He has been a senior researcher in the Agency for Defense Development (ADD) from 2012 to now. His research interests include heterogeneous communication systems, and neural networks for microwave and optical systems. 


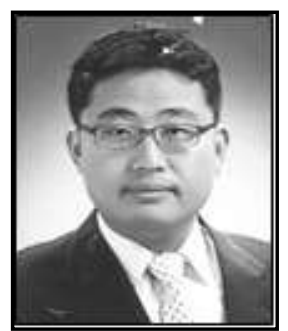

Ju-Hang Sohn, he received M.S. degree in Computer Science from Soongsil University, Seoul, Korea, in 2005. From 2005 to 2007 and from 2010 to 2012, he worked as a researcher at ETRI (Eletronics and Telecommunications Research Institute). From 2007 to 2010, he worked as a researcher at ADD (Agency for Defense Development). He has been a senior researcher in the Agency for Defense Development (ADD) from 2012 to now. His research interests include network topology, M\&S, wireless telecommunications. 